\title{
Software-guided microscale flow calorimeter for efficient acquisition of thermokinetic data
}

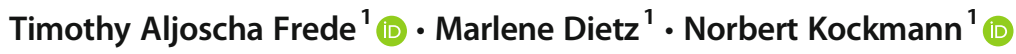 \\ Received: 14 December 2020 / Accepted: 8 February 2021 / Published online: 15 March 2021 \\ (C) The Author(s) 2021
}

\begin{abstract}
Fast chemical process development is inevitably linked to an optimized determination of thermokinetic data of chemical reactions. A miniaturized flow calorimeter enables increased sensitivity when examining small amounts of reactants in a short time compared to traditional batch equipment. Therefore, a methodology to determine optimal reaction conditions for calorimetric measurement experiments was developed and is presented in this contribution. Within the methodology, short-cut calculations are supplemented by computational fluid dynamics (CFD) simulations for a better representation of the hydrodynamics within the microreactor. This approach leads to the effective design of experiments. Unfavourable experimental conditions for kinetics experiments are determined in advance and therefore, need not to be considered during design of experiments. The methodology is tested for an instantaneous acid-base reaction. Good agreement of simulations was obtained with experimental data. Thus, the prediction of the hydrodynamics is enabled and the first steps towards a digital twin of the calorimeter are performed. The flow rates proposed by the methodology are tested for the determination of reaction enthalpy and showed that reasonable experimental settings resulted.
\end{abstract}

Keywords Continuous reaction calorimetry $\cdot$ Flow chemistry $\cdot$ CFD simulation $\cdot$ Microreaction technology $\cdot$ Design of experiments · Digital twin

\section{Introduction}

Continuously operated processes feature higher yields and selectivity, facilitate process automation, reduce the ecological footprint and offer shorter process development times [1-3]. For the development of accurate kinetic models that assist chemical reactor development, design and optimization, extensive knowledge of interaction between reaction kinetics and hydrodynamics is essential [4]. Thus, the interest in the

\section{Highlights}

- Development of a methodology for selecting suitable kinetics experiments.

- Good agreement of simulations with experimental data validates the CFD model.

- Number of possible experimental settings has been halved.

Timothy Aljoscha Frede

Timothy.Frede@tu-dortmund.de

1 Department of Biochemical and Chemical Engineering, Laboratory of Equipment Design, TU Dortmund University, Emil-Figge-Straße 68, 44227 Dortmund, Germany development of continuous flow calorimeter increases constantly [5-8]. Moreover, the miniaturization of calorimetry enables the investigation of fast and highly exothermic reactions under safe conditions due to the superior temperature control when compared to standard batch equipment [9]. Traditionally, thermokinetic data is experimentally obtained during process development using mostly batch equipment. These studies provide reliable information about the physical processes. Yet, only limited information is obtained about the dynamic behavior within the reactor performing those experiments. Efficiency and effectiveness of process development can be increased by digitalization of research and development [10]. The systematical use of simulation tools such as computational fluid dynamics (CFD) is essential to overcome challenges related to experimental measurements and to optimize real processes $[10,11]$. Thus, information regarding transient flow behavior, velocity, pressure and concentration fields is gained and can be used to reduce the number of experiments. Once the model has been validated, process performance can be predicted under different flow conditions, reactant concentrations and reactor configurations [11, 12]. The efficient combination of CFD and experiments for 
evaluation of microreactor performance for fast and exothermic reactions has already been shown by Asano et al. [13].

In this work, a methodology is developed for the selection of experiments in a continuous flow calorimeter during chemical process development aiming a time and cost-efficient acquisition of thermokinetic data. The methodology enables choosing the optimal reaction conditions out of the entire possible range of experimental parameters of the calorimeter rather than performing full factorial design of experiment. Thus, optimal designs of experiments are determined, which offer maximal information gain with minimal effort. Finally, the proposed experimental settings are tested for the determination of the reaction enthalpy of a test reaction.

\section{Materials and Methods}

\section{Calorimeter and experimental setup}

The calorimeter's setup and its peripheral equipment have been described in previous works of Reichmann et al. [14, 15]. A central programmable logic controller (PLC) (LabManager ${ }^{\circledR}$, HiTec Zang GmbH, Germany) has been added to the experimental setup to automate the execution of calibration and experiments. The current setup and the employed microreactor (type*-S, HTM series, Little Things Factory $\mathrm{GmbH}$, Germany) are displayed in Fig. 1. Compared to the setup in [15], only four Peltier elements are used here, whose projection area is about 4.5 times larger than the previously used ones. The microreactor is made of glass and features a Y-mixer to contact the incoming fluids and 19 chicane mixers to improve mixing. The hydraulic diameter at the smallest cross-sectional area within the reactor is $1 \mathrm{~mm}$, which is used for the subsequent calculations.

The technical data of the employed microreactor and syringe pumps is given in Table 1 .

\section{Evaluation of optimal reaction conditions}

The methodology focusses on the evaluation of optimal reaction conditions, which are suited for continuous calorimetric measurement experiments. The basis for this methodology is the approach of Krasberg et al. [16], who developed a methodology to select plug flow equipment for a modular and continuous small-scale plant. A schematic overview of the stepwise methodology is shown in Fig. 2.

The methodology includes CFD simulations complemented by short-cut methods. The methodology is a stepwise approach, in which the demand of data increases with the sequence of the steps. However, the possibility to restrict the experimental design space is also increasing. In the following, the available information is described with the individual steps of the methodology.

\section{Input data}

The input data sets represent the basis for the subsequent steps. In this study, reaction conditions are proposed based on the

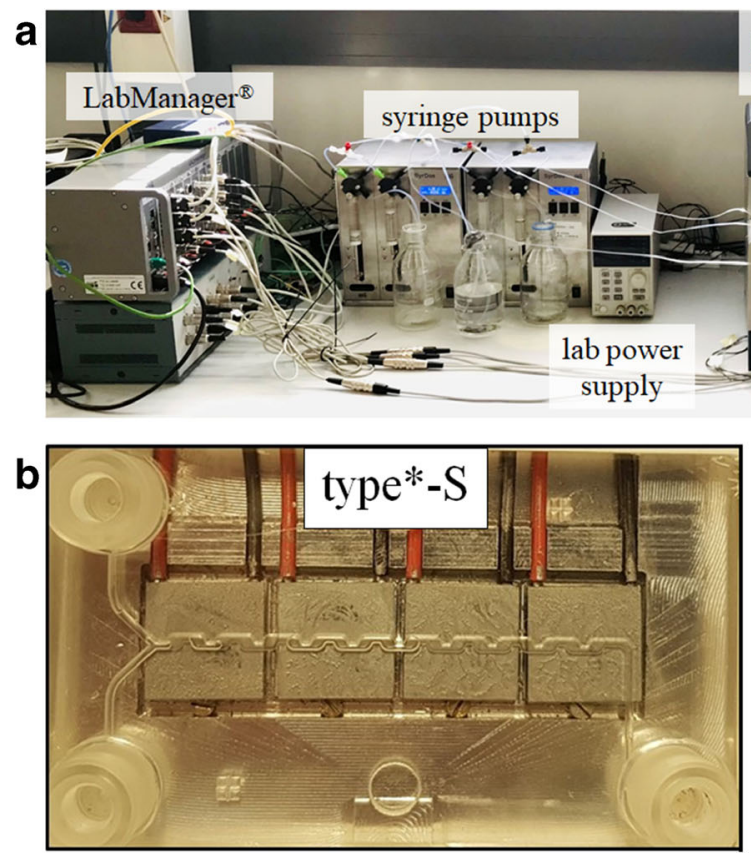

Fig. 1 a Experimental setup for continuous reaction calorimetry consisting of laboratory automation system (LabManager $\left.{ }^{\circledR}\right)$, syringe pumps for feeding, preheating thermostat, calorimeter itself with a gear pump and thermostat for tempering the base plate. $\mathbf{b}$ Microreactor within the reaction calorimeter with four Peltier elements $\left(15 \times 15 \mathrm{~mm}^{2}\right)$, which are positioned on the base plate 
Table 1 Technical data of the microreactor and the pumps used

\begin{tabular}{|c|c|c|c|c|c|}
\hline device & data type & symbol & unit & value & comment \\
\hline \multirow[t]{2}{*}{ type*-S } & geometries & $V_{\mathrm{R}}$ & $\mathrm{mL}$ & 0.10 & \\
\hline & & $d_{\mathrm{h}}$ & $\mathrm{mm}$ & 1.00 & $d_{\mathrm{h}}=d_{\mathrm{i}}$ \\
\hline SyrDos $^{\mathrm{TM}} 2$ & possible flow rates & $\begin{array}{l}\dot{V}_{\min } \\
\dot{V}_{\max }\end{array}$ & $\begin{array}{l}\mathrm{mL} \min ^{-1} \\
\mathrm{~mL} \min ^{-1}\end{array}$ & $\begin{array}{l}0.5 \\
6.00\end{array}$ & $\begin{array}{l}\text { limited by } \\
\text { syringes used in } \\
\text { the pumps }\end{array}$ \\
\hline
\end{tabular}

chemical reaction to be investigated and the used microreactor. For the chemical reaction system, information regarding the physical properties of the reaction mixture in terms of density, viscosity and diffusivity is required or has to be estimated. In addition, a rough classification of the reaction kinetics supports the methodology. The microreactors are defined regarding their geometries and allowed operation conditions. Since commercially available microreactors are employed, this information is provided by the manufacturer.

\section{Step 1: Residence time}

The residence time of fluids within microstructured devices is in the order of seconds or less, which can be detrimental to slow reactions [17]. Thus, the residence time is a crucial factor for the successful investigation of chemical reactions in continuously operated reactors. In case of insufficient residence time, complete conversion cannot be guaranteed, which reduces the quality of the information gained during calorimetric measurement. Otherwise, excessive residence times and broad residence time distributions promote side or subsequent reactions, which negatively influence the selectivity and yield. The hydraulic residence time $\tau_{\mathrm{i}}$ is calculated dividing the reaction volume $V_{\mathrm{R}}$ by the volumetric flow rate $\dot{V}$, shown in Eq. 1 .

$\tau_{\mathrm{i}}=\frac{V_{\mathrm{R}}}{\dot{V}}$

The range of possible volumetric flow rates is determined by the pumps used in the experimental setup. The reactor volume is obtained from the microreactor's geometry.

\section{Step 2: Pressure drop}

The pressure drop represents a technical criterion. Among others, the reactor itself and the pumps limit the maximum permissible pressure loss. The pressure is expected to be highest at the reactor inlet. The pressure drop can be calculated using generalized models, as shown in Eq. 2 [18].

$\Delta p_{\mathrm{i}}=\left(\lambda_{\mathrm{i}} \frac{l_{\mathrm{i}}}{d_{\mathrm{h}, \mathrm{i}}}+\omega_{\mathrm{i}}\right) \cdot \frac{\bar{\rho}}{2} \bar{w}_{\mathrm{i}}^{2}$

However, the friction factor $\lambda_{\mathrm{i}}$ and the influence of secondary flow patterns $\omega_{i}$ have to be determined for each microreactor. For this purpose, microreactors are characterized using semi-empirical pressure drop modeling [19]. The Dean number $D n$ is used to characterize the influence of secondary flow profiles which are the result of centrifugal forces. In Eq. 3, the channel's curvature is expressed by $d_{c}$. [20]

$D n=\operatorname{Re} \sqrt{\frac{d_{\mathrm{h}}}{d_{\mathrm{c}}}}$

In order to improve the determination of the pressure drop and later the mixing performance, the microreactor's hydrodynamics including the pressure drop are estimated using CFD simulations. Steady state CFD simulations are carried out for incompressible, single-phase flow through the microreactor using the open source software OpenFOAM. The solver icoFoam is used to investigate the hydrodynamics, which is completed by a passive scalar for solute transport. The data obtained is analyzed regarding the pressure drop as a function of volumetric flow rate.
Fig. 2 Methodology to evaluate optimal reaction conditions for efficient acquisition of kinetic data, where the experimental design space is limited by the stepwise determination of important time scales based on specified input data

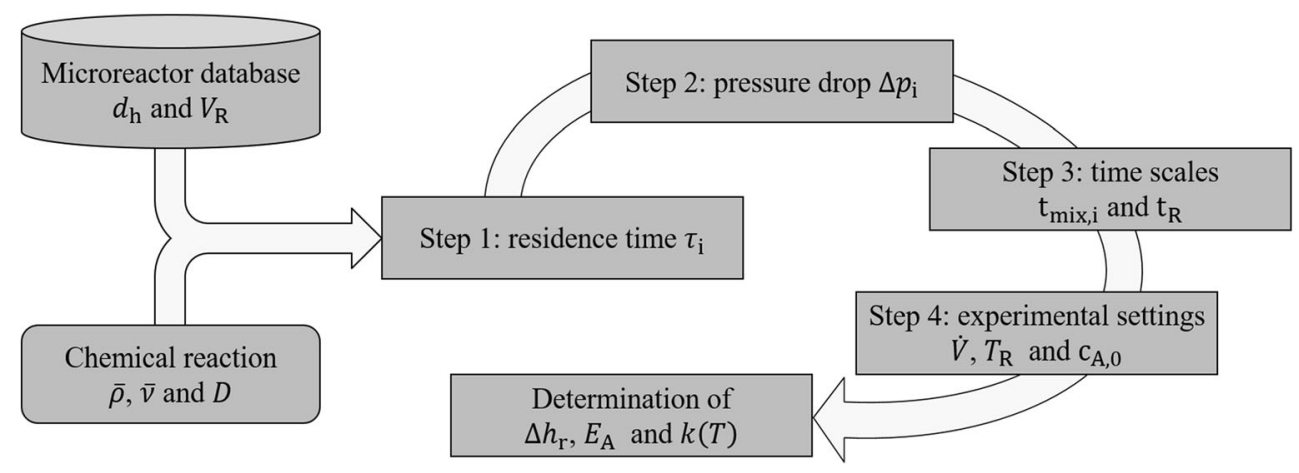




\section{Step 3: Time scales}

Besides the residence time, mixing of reactants and its corresponding time scale are decisive during chemical transformation $[21,22]$. Thus, the determination of mixing time scales is of great interest, especially in different microreactors. Theoretically, laminar flow prevails in microreactors, since $R e<2300$ [23]. However, secondary flow patterns such as Dean flow and engulfment are found in bend channels for $R e>100$, which greatly enhance mixing [9, 24, 25]. A theoretical mixing time can be estimated roughly using a short-cut method considering micromixing by engulfment [26]. For this purpose, a power law relationship between energy dissipation rate and mixing time was presented by Falk and Commenge [27]. The mixing time depends on the mean kinematic viscosity of the reaction medium $\bar{\nu}$, the energy dissipation rate $\epsilon_{\mathrm{i}}$ and a pre-factor matching the engulfment theory, shown in Eq. 5. The energy dissipation rate is defined in Eq. 6 with volumetric flow rate $\dot{V}_{\text {tot }}$, pressure drop $\Delta p_{i}$, the mean fluid's density $\bar{\rho}$ and the dissipation volume $V_{\mathrm{i}}$. The pressure drop for these calculations is obtained from CFD simulations in step 2 .

$$
\begin{aligned}
& R e=\frac{d_{\mathrm{h}} \bar{w}}{\bar{\nu}} \\
& t_{\text {mix }, \mathrm{i}}=17.3\left(\frac{\bar{\nu}}{\epsilon_{\mathrm{i}}}\right)^{1 / 2} \\
& \epsilon_{\mathrm{i}}=\frac{\Delta p_{\mathrm{i}} \dot{V}}{\bar{\rho} V_{\mathrm{i}}}
\end{aligned}
$$

Besides the estimation using the short-cut method, the mixing performance of the microreactor is characterized using the CFD model. Therefore, the variance of the concentration profile of the solute is evaluated at several cross sections of the mixing channel. Since the flow velocity varies over the cross section, the velocity-weighted mixing quality $\alpha_{\dot{V}}$ is used for a better representation of the flow situation, shown in Eq. 7. In Eq. 8, the concentration $c_{1}$ at a grid point is weighted with the velocity $w_{\mathrm{i}}$ at this grid point, and the mean velocity $\bar{w}$ with the cross section $A_{\mathrm{M}}$. Based on the position of complete mixing, the corresponding channel length $l_{\mathrm{i}}$ and the mixing time $t_{\text {mix,CFD }}$ can be determined by dividing the obtained channel length with the mean velocity, shown in Eq. 10. [28]

$$
\begin{aligned}
& \alpha_{\mathrm{V}}=1-\sqrt{\frac{\sigma_{\dot{\mathrm{V}}}^{2}(c)}{\sigma_{\dot{\mathrm{V}}, \text { max }}^{2}(c)}} \\
& \sigma_{\dot{\mathrm{V}}}^{2}(c)=\frac{1}{A_{\mathrm{M}} \bar{w}} \int_{A_{\mathrm{M}}}\left(c_{i}-\frac{\int_{A_{\mathrm{M}}} c_{\mathrm{i}} w_{\mathrm{i}} d A}{\int_{A_{\mathrm{M}}} w_{\mathrm{i}} d A}\right)^{2} w d A
\end{aligned}
$$

$$
\begin{aligned}
& \sigma_{\mathrm{V}, \text { max }}^{2}(c)=\frac{\dot{V}_{1} \dot{V}_{2}}{\left(\dot{V}_{1}+\dot{V}_{2}\right)^{2}}\left(c_{1}-c_{2}\right)^{2} \\
& t_{\text {mix }, \mathrm{CFD}}=\frac{l_{\mathrm{i}}}{\bar{w}}
\end{aligned}
$$

Besides the mixing time scale, the characteristic reaction time is an important quantity in process engineering [28]. The reaction time can be estimated using the reaction order $m$, the starting concentration of the limiting component $c_{\mathrm{A} 0}$ and the reaction rate constant $k$, which is influenced by temperature, shown in Eq. 11.

$t_{\mathrm{R}}=\frac{1}{k\left(T_{\mathrm{R}}\right) \cdot c_{\mathrm{A} 0}^{m-1}}$

Since kinetic data is not available at this point, an educated guess based on similar reactions or heuristics for classification of the reaction can be used to estimate the reaction rate constant. A classification of the reaction according to Roberge [29] and Kashid et al. [9] allows for roughly estimating the reaction time. However, the educatedguess based experience and intuition play an eminent role at this stage of process development. In this case, the methodology has to be started with the operator assuming certain kinetic data. Since the goal of the method is initially only to stake out the experimental design space, a rough estimate is sufficient for now. Based on this data, short test trials must be performed to check whether complete conversion is achieved in the reactor. If this is not the case, the constraint for the reaction time must be adjusted iteratively. The exact kinetic data will then be determined by the experiments and subsequently, CFD simulations can be performed with the experimentally determined data to validate the model for future applications. For optimal reaction conditions and consequently optimal results, the three characteristic times of residence $\tau_{i}$, mixing $t_{\text {mix,i }}$ and reaction $t_{\mathrm{R}}$ have to be designed properly. Complete conversion within the microreactor can be assumed, if both the mixing and reaction time are faster than the mean residence time. Furthermore, two regimes can be distinguished. In a reaction-dominated regime, mixing time is faster than reaction time, while in a mixing-dominated regime, it is opposite. Experimental settings are rated as suitable if the arrangement of the characteristic times follows the proposed sequence.

\section{Experimental settings}

The resulting constraints regarding the reaction and flow conditions are visualized within a parameter plot to determine suitable experimental settings, which is shown exemplarily in Fig. 3 


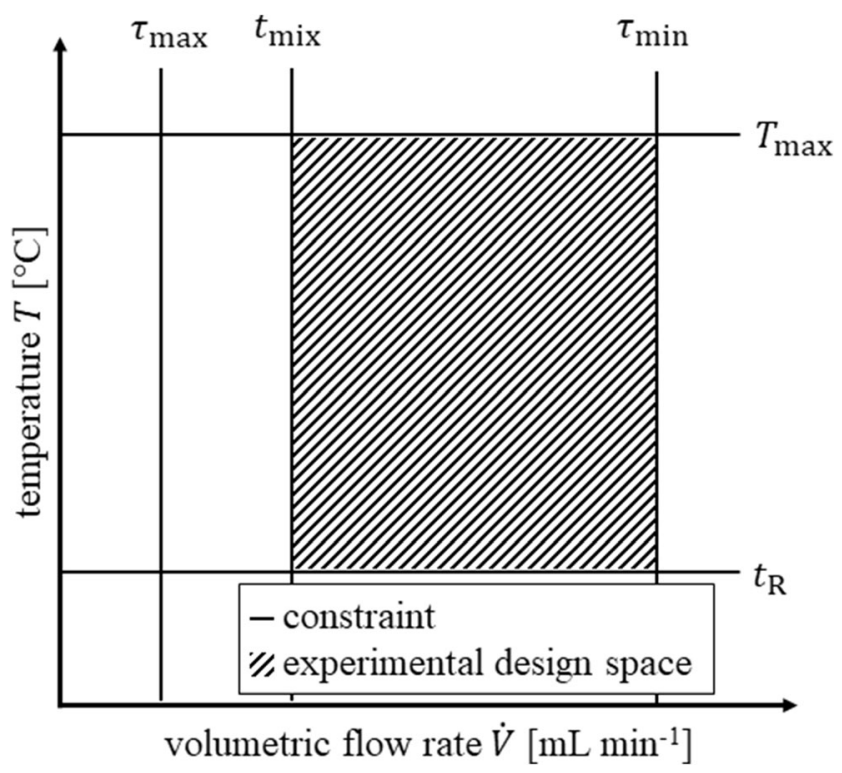

Fig. 3 Qualitative representation of the experimental design space to evaluate suitable experimental settings for calorimetric measurements as function of temperature and volumetric flow rate. The constraints result from the steps of the presented methodology.

Three conditions are essential to span the experimental design space:

- ranges and discrete step sizes of the experimental parameters

- values for operating constraints

- values of the constraints as a function of the experimental parameters

Consequently, suitable experimental settings are located inside the constraints. The resulting settings are translated into control commands for the calorimeter, e.g. into settings of the pumping system to adjust the volumetric flow rate to achieve a specific residence time. Therefore, the experimental settings are transferred via Open Platform Communications (OPC) Unified Architecture (UA) to the PLC, which controls the whole experimental setup.

\section{Determination of thermokinetic data}

In this step, the control commands for the calorimeter are executed automatically using the PLC and subsequently, the measured values are evaluated. Currently, the calorimeter is operated in isoperibolic mode. The calorimetric measurement method has been described by Reichmann et al. [14] and is used here to determine the reaction enthalpy.

\section{Case Study}

A well-known, exothermic reaction from the literature - acidbase reaction of hydrochloric acid $(\mathrm{HCl})$ and sodium hydroxide $(\mathrm{NaOH})$ - is used to demonstrate the functionality of the presented methodology. Sen et al. [30] also investigated this model reaction for calorimetry using CFD for the development of a microfluidic reaction calorimeter. The thermokinetic parameters, which were determined experimentally, are given in Table 2. For nearly equimolar conditions, the neutralization reaction follows a second-order kinetics. [31]

$\mathrm{H}^{+} \mathrm{Cl}^{-}+\mathrm{Na}^{+} \mathrm{OH}^{-} \rightarrow \mathrm{H}_{2} \mathrm{O}+\mathrm{Na}^{+} \mathrm{Cl}^{-}$

Since aqueous solutions of $\mathrm{HCl}$ and $\mathrm{NaOH}$ with concentrations of $1.0 \mathrm{M}$ and $1.1 \mathrm{M}$ respectively are used in this study, the physical properties of water are used in the short-cut calculations and in the advanced CFD calculations. The binary diffusion coefficient $D$ is obtained from Dortmund Data Bank with a value of $9.6 \cdot 10^{-10} \mathrm{~m}^{2} \mathrm{~s}^{-1}$.

\section{Results and discussion}

In this section, the results of the performance of the individual steps are presented to determine suitable experimental settings for the investigation of the neutralization reaction. Subsequently, the obtained reaction conditions are tested and the CFD model is validated using this experimental data.

\section{Case study, step 1: Residence time}

At first, the possible range of residence time is checked for the investigated reactor and syringe pumps used in the experimental setup. According to Eq. 1 and Table 1, the combination of microreactor and pumps provides a residence time between $\tau_{\text {min }}=0.5 \mathrm{~s}$ and $\tau_{\max }=6.0 \mathrm{~s}$.

\section{Case study, step 2: Pressure drop}

The hydrodynamics in the reactor, and thus the pressure drop, too, are calculated on the basis of CFD simulations. Pressure drops within the microreactor are displayed in Fig. 4 for varying volumetric flow rates.

In general, the pressure drop increases for higher volumetric flow rates. A second-order polynomial, as described by the Darcy-Weisbach equation, matches the data according to Eq. 2 with the highest coefficient of determination of $R^{2}=1$.

Table 2 Kinetic data of neutralization reaction of hydrochloric acid and sodium hydroxide. [31]

\begin{tabular}{llll}
\hline $\mathrm{k}_{0}\left[\mathrm{~L} \mathrm{~mol}^{-1} \mathrm{~s}^{-1}\right]$ & $\mathrm{E}_{\mathrm{A}}\left[\mathrm{J} \mathrm{kmol}^{-1}\right]$ & $\Delta \mathrm{h}_{\mathrm{r}}\left[\mathrm{kJ} \mathrm{mol}^{-1}\right]$ & $\mathrm{m} \mathrm{[-]}$ \\
$1.69 \cdot 10^{11}$ & $3.33 \cdot 10^{6}$ & -57.6 & 2 \\
\hline
\end{tabular}




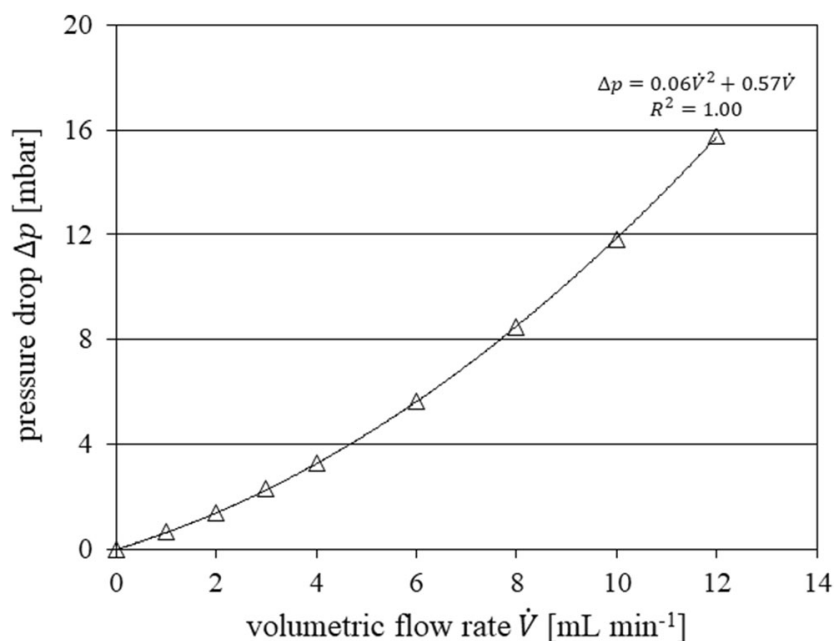

Fig. 4 Simulated values for pressure drop over volumetric flow rates for the type*-S microreactor (HTM series, Little Things Factory GmbH)

The pressure drop ranges from 0.07 to $15.78 \mathrm{mbar}$, corresponding to volumetric flow rates of $1-12 \mathrm{~mL} \mathrm{~min}^{-1}$, flow velocities of 21.22 to $254.65 \mathrm{~mm} \mathrm{~s}^{-1}$ and Reynolds numbers of 23 to 285, respectively. Pressure loss caused by piping, which was not considered within the CFD simulations, was estimated using Hagen-Poiseuille equation and resulted in a maximum loss of $0.2 \mathrm{mbar}$ for the highest possible flow rate. Hence, this pressure loss is negligible compared to the loss induced by the reactor.

\section{Case study, step 3: Times scales}

At first, the energy dissipation rates are calculated based on the CFD results and displayed for varying flow rates in Fig. 5.

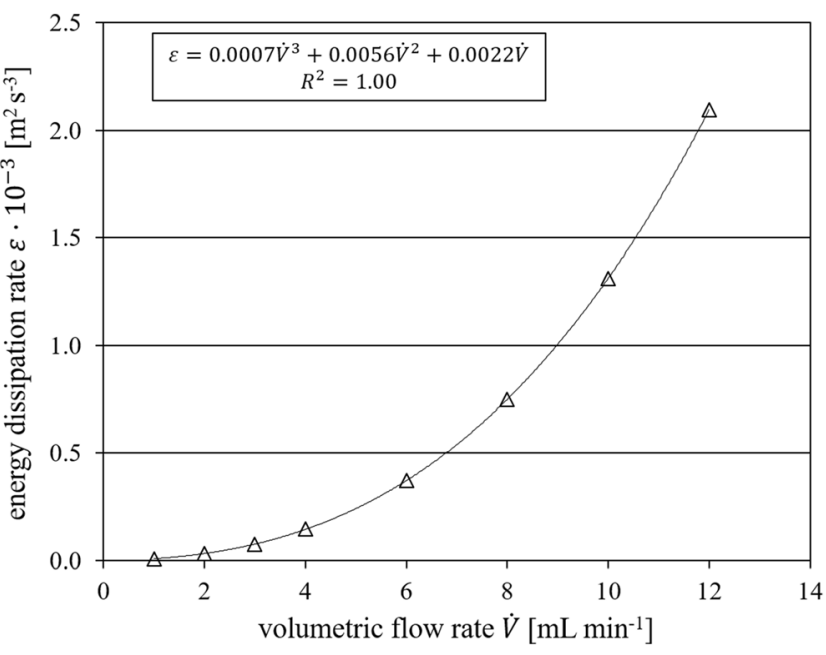

Fig. 5 Energy dissipation rates over volumetric flow rates based on CFD results for the type* $-\mathrm{S}$ microreactor (HTM series, Little Things Factory $\mathrm{GmbH})$

The energy dissipation rate increases with higher flow rates. For higher flow rates, the cubic relationship between energy dissipation and volumetric flow rate becomes apparent, as the velocity enters Eq. 6 to the power of three. Subsequently, the velocity-weighted mixing quality is determined at various cross sections within the microreactor for the respective flow rates, shown in Fig. 6. The cross sections are located in the middle of the straight part behind the chicane mixer.

In general, the mixing quality increases with increasing distance from the reactor inlet and with increasing flow rate. Additionally, three distinct regimes can be observed. For
Fig. 6 a Allocated position of cross sections within the microreactor for the evaluation of the mixing quality. b Velocityweighted mixing quality over allocated position of cross sections within the microreactor for varying volumetric flow rates c Concentration profile of $\mathrm{NaOH}$ for $V=8 \mathrm{~mL} \mathrm{~min}^{-1}$ in the type*$\mathrm{S}$ microreactor (HTM series, Little Things Factory $\mathrm{GmbH}$ ). Simulations using icoFoam, postprocessing in ParaView.
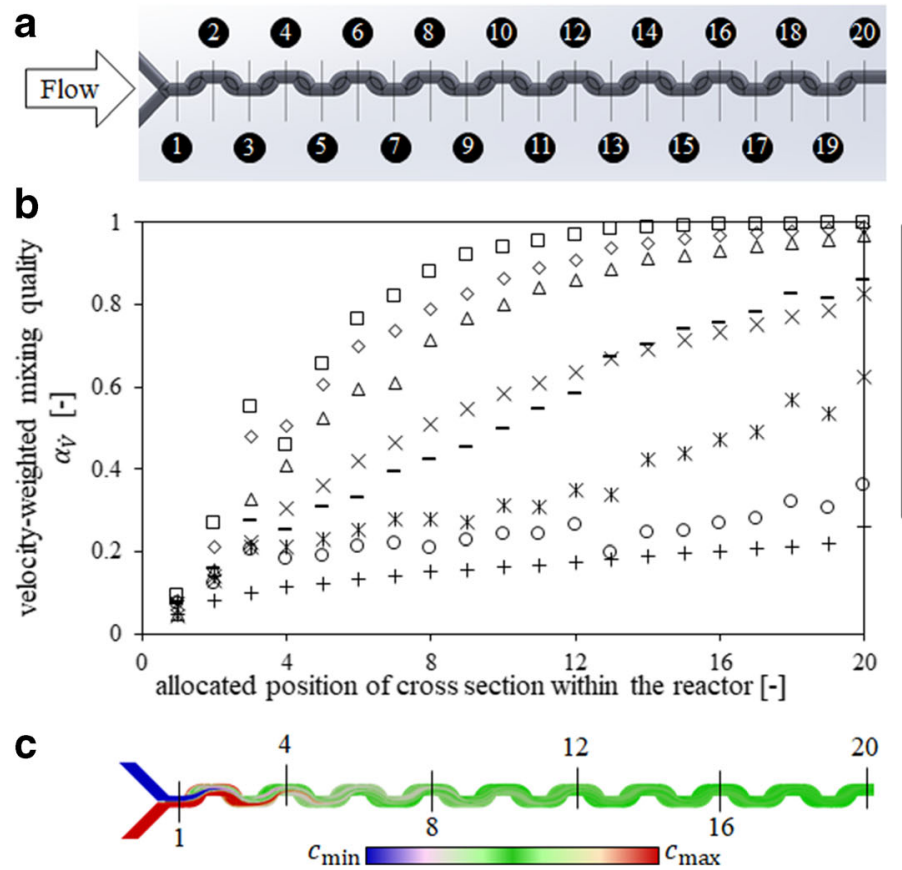

$\square 12 \mathrm{~mL} \mathrm{~min}^{-1}$ $\triangle 10 \mathrm{~mL} \mathrm{~min}^{-1}$ $\diamond 8 \mathrm{~mL} \mathrm{~min}^{-1}$ $\times 6 \mathrm{~mL} \mathrm{~min}^{-1}$ - $4 \mathrm{~mL} \mathrm{~min}^{-1}$ * $3 \mathrm{~mL} \mathrm{~min}^{-1}$ O $2 \mathrm{~mL} \mathrm{~min}^{-1}$ $+1 \mathrm{~mL} \mathrm{~min}^{-1}$ 
Table 3 Positions and corresponding channel lengths of complete mixing derived from CFD simulations

\begin{tabular}{lll}
\hline$V\left[\mathrm{~mL} \mathrm{~min}{ }^{-1}\right]$ & Position $[-]$ & Channel length $[\mathrm{mm}]$ \\
\hline$<6$ & - & - \\
8 & 14 & 52.98 \\
10 & 19 & 72.98 \\
12 & 11 & 40.98 \\
\hline
\end{tabular}

- indicates no complete mixing within the reactor

$\dot{V} \leq 3 \mathrm{~mL} \min ^{-1}$, the mixing quality increases almost linearly along the reactor coordinate. In this regime, the Reynolds and the corresponding Dean number are comparatively small ( $R e \leq 75$ and $D n \leq 50)$ and mixing is assumed to be dominated by diffusion. Hence, the maximum achieved mixing quality $\alpha_{\dot{V}}$ remains below 0.6 . However, the slope of the mixing quality increases significantly for 4 and 6 $\mathrm{mL} \mathrm{min}^{-1}$. For these volumetric flow rates, the Dean number takes values of 67 and 100 respectively. Thus, secondary flow patterns can be assumed, which lead to improved convective mixing. According to Ligrani [32], Dean vortices are expected to be fully developed and stable for $D n>64$ and thus, mixing is greatly enhanced. This can also be derived from the higher mechanical energy consumption, which can be seen in the energy dissipation rate graph. For $\dot{V} \geq 8 \mathrm{~mL} \mathrm{~min}^{-1}$, the course of mixing quality is almost identical. $R e$ and $D n$ are relatively high with $R e \geq 190$ and $D n \geq 134$. For $R e>150$, the mixing performance of chicane mixers is significantly enhanced due to stable vortex generation and mixing quality increases noticeably [33]. Based on these results, complete mixing within the reactor is assumed for $\dot{V} \geq 8 \mathrm{~mL} \mathrm{~min}^{-1}$.

Subsequently, the mixing time is calculated based on the results of the mixing performance. For this purpose, a limit must be set for the mixing quality at which the mixture is considered completely mixed for the microreactor used in this work. As can be seen in Fig. 6b, a plateau of the mixing quality is reached within the reactor only for $\dot{V} \geq 8 \mathrm{~mL} \mathrm{~min}^{-1}$. For $\dot{V} \leq 6 \mathrm{~mL} \mathrm{~min}^{-1}$, the mixing quality continues to increase along the reactor channel and does not reach an almost constant value. Based on this observation, the limit for the mixing quality is set so that complete mixing is only assumed for the last three volume flows. Thus, complete mixing within the reactor is assumed from the cross-sectional area at which a limiting value of $\alpha_{V} \geq 0.95$ is reached. The positions and corresponding channel lengths of complete mixing are given in Table 3. The results presented are in good agreement with the study of Khaydarov et al. [34] on a reactor with the same mixing structure. Especially the presented flow regime could also be observed here at the investigated Reynolds numbers.

Table 3 shows that the channel length, at which complete mixing is achieved, does not consistently decrease with increasing volume flow. This observation is due to the flow behavior at different flow rates. Figure 7 illustrates the streamlines within the first nine chicane mixers.

According to Fig. 7, the vortex regime is observed for the displayed flow rates. However, a slightly different flow behavior is evident between $\dot{V}=8 \mathrm{~mL} \mathrm{~min}^{-1}$ and $\dot{V}=10 \mathrm{~mL} \mathrm{~min}^{-1}$. For $\dot{V}=8 \mathrm{~mL} \mathrm{~min}^{-1}$, the streamlines in the middle of the channel are stronger, which can be seen in a darker coloring. Thus, mixing in this area is improved compared to the flow behavior of $\dot{V}=10 \mathrm{~mL} \mathrm{~min}^{-1}$. The renewed increase in mixing quality for $\dot{V}=12 \mathrm{~mL} \mathrm{~min}^{-1}$ is due to the stronger expression of the vortices within the recirculation zones.

Figure 8 displays the mixing times plotted over the corresponding energy dissipation rate using the short-cut method and the CFD model.
Fig. 7 Simulated streamlines for different volumetric flow rates of 8, 10 and $12 \mathrm{~mL} \mathrm{~min}^{-1}(\operatorname{Re}=190$, 237 and 285). Postprocessing in ParaView

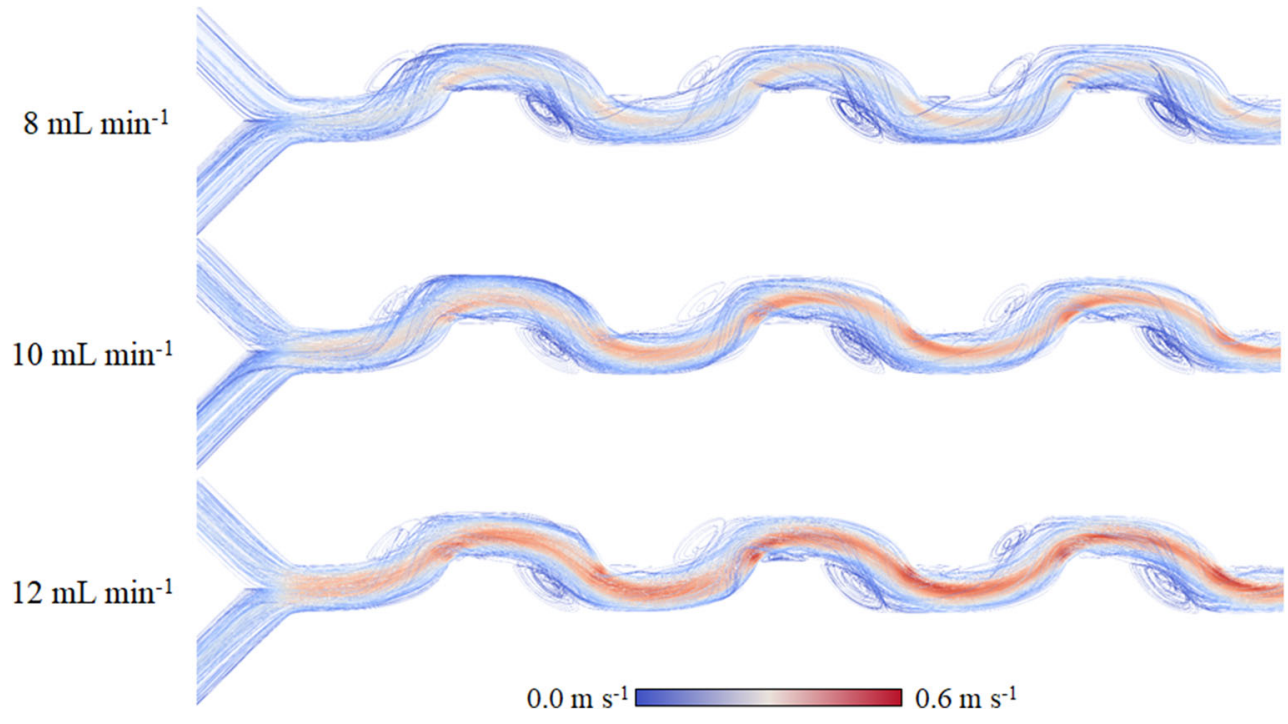




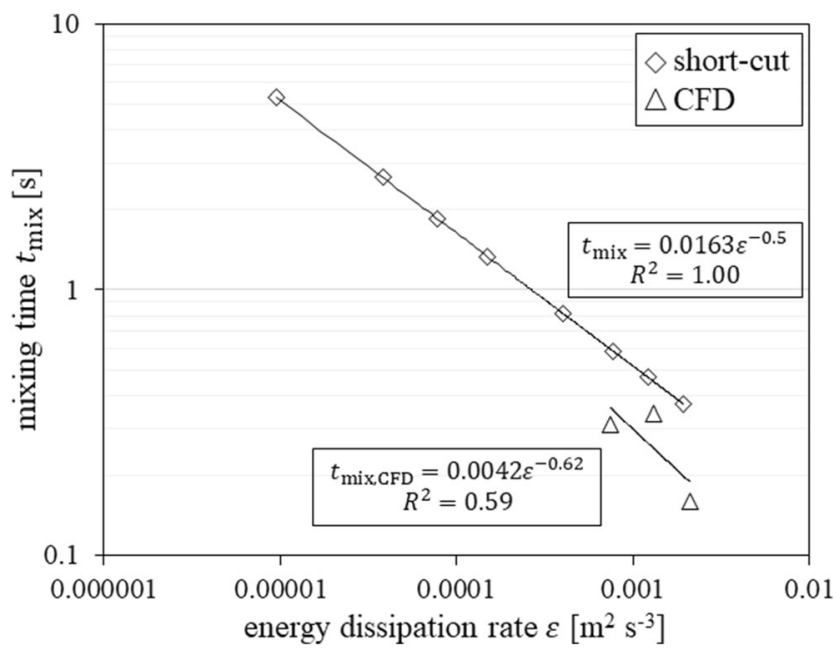

Fig. 8 Mixing time scale plotted over energy dissipation rates with power law trend line that fits the data of the short-cut method and the CFD simulations

In general, mixing times decrease with increasing energy dissipation rates. Furthermore, the values of both calculations are in the same order of magnitude for the respective flow rates. Using the short-cut method, the mixing times are between 0.37 and $5.28 \mathrm{~s}$ and those of the CFD simulations between 0.16 and $0.34 \mathrm{~s}$. For the CFD results, the coefficient of determination shows a relative low value with 0.59 for the data fitting power law trend line. Additionally, the exponent with a value of $n=0.62$ differs from the theoretical value of $n=$ 0.5 [27]. The deviations between the CFD simulations and the literature are probably due to the setting of the limiting value for complete mixing. Moreover, the need for a detailed characterization of the hydrodynamics is illustrated by the fact that the short-cut calculates mixing times for each flow rate, whereas the simulations show that complete mixing cannot be achieved for $\dot{V} \leq 6 \mathrm{~mL} \mathrm{~min}{ }^{-1}$.

Subsequently, the characteristic reaction time of the neutralization reaction is calculated. According to Eq. 11 and the kinetic data from Table 2, the reaction time $t_{\mathrm{R}}$ is $2.6 \cdot 10^{-11} \mathrm{~s}$. This is consistent with the literature, as the ion reaction of a proton and a hydroxide ion to form a water belongs to quasiinstantaneous reactions [35]. Since the reaction time will always be shorter than the mixing time in this case, a mixingdominated regime prevails. If reaction time exceeded the residence time, the selected reactor would not be suitable for the investigated reaction. In this case, another reactor needs to be selected from the database, which, depending on the limitation, either mixes faster or offers more residence time. Figure 9 displays the mixing and residence time over volumetric flow rates.

Figure 9 shows that the time scales are designed properly only for $\dot{V} \geq 8 \mathrm{~mL} \mathrm{~min}{ }^{-1}$. Therefore, only with these settings, complete conversion can be expected and thus the information content is at its maximum.

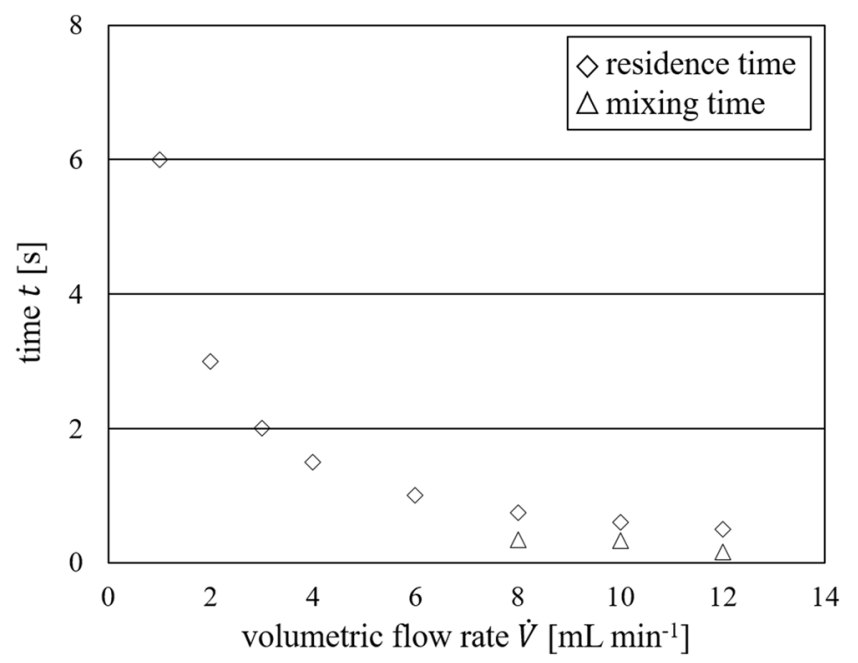

Fig. 9 Residence and mixing time over volumetric flow rates

\section{Model validation}

In order to validate the CFD results, the data obtained is compared with experimental measurements. The pressure was measured at reactor inlet and outlet via a T-shaped fitting using pressure sensors (type A-10, WIKA Alexander Wiegand SE \& Co. KG, Germany). In Fig. 10, the pressure drop is plotted over the volumetric flow rate.

As observed, the pressure drop obtained with CFD is in good agreement with the experimental pressure drop.

In a next step, the CFD and experimental mixing times are compared. Experimental mixing times were determined optically and from heat flux profiles by Reichmann et al. [15]. The mixing times for different volumetric flow rates are shown in Fig. 11.

In general, the mixing time obtained with CFD is in a relatively good agreement with the experimental mixing time. However, a larger deviation of $0.16 \mathrm{~s}$ can be seen for $\dot{V}=10 \mathrm{~mL} \mathrm{~min}{ }^{-1}$, which has been discussed on the

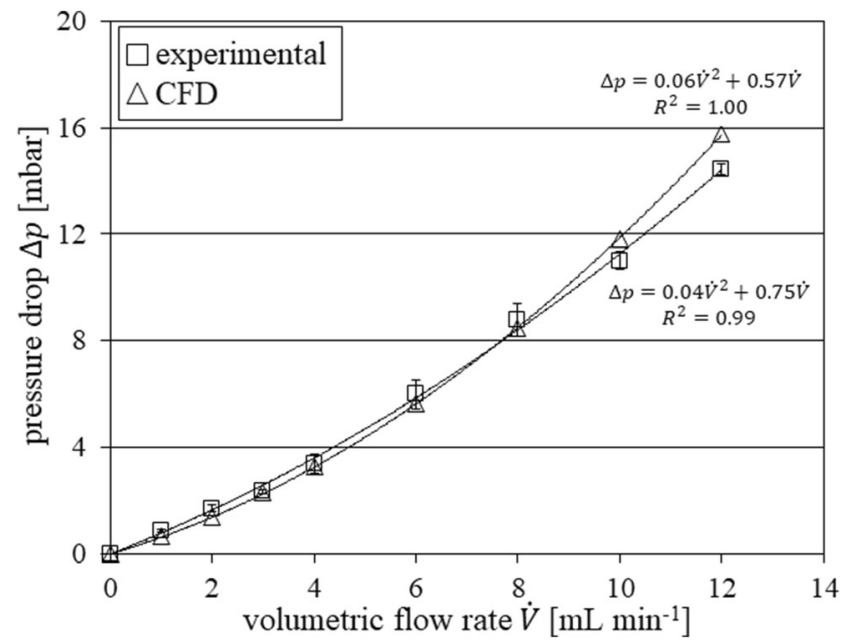

Fig. 10 Comparison of CFD and experimental pressure drop over volumetric flow rates 


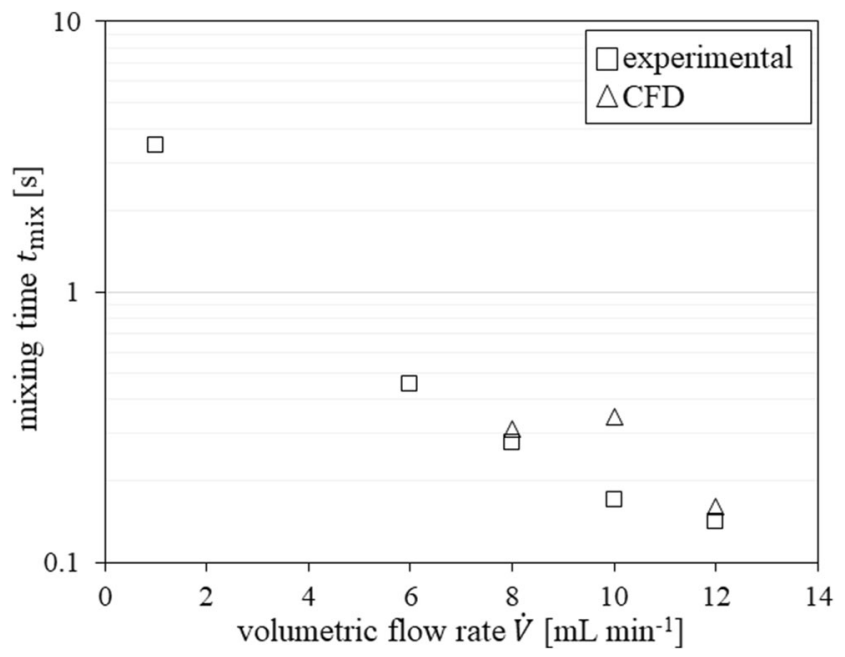

Fig. 11 Comparison of CFD and experimental mixing time over volumetric flow rates

basis of the flow behavior. For $\dot{V}=6 \mathrm{~mL} \mathrm{~min}^{-1}$, no complete mixing within the reactor is calculated by CFD. Moreover, complete mixing was also observed experimentally for $\dot{V}=1 \mathrm{~mL} \min ^{-1}$ with a mixing time of a $3.57 \mathrm{~s}$. Due to the low Reynolds number $(R e=23)$, mixing is dominated by diffusion. However, diffusive mixing is supported by convective mixing as theoretical diffusion time is $12.5 \mathrm{~s}$ [15]. The deviation can be attributed to the flow regime and the assumed diffusion coefficient. On the one hand, the streamlines between CFD and experiment may differ slightly, resulting, for example, in different dead volumes and thus longer residence times than in the real reactor. On the other hand, the diffusion coefficient may be too low, since it was used for pure water. Although the aspects have only a minor influence on their own, the interaction of both can influence the results.

Since this mixing time is not covered by the CFD model, it is to be expected that the interaction of diffusive and convective mixing is not yet sufficiently described by the simulations, especially for very small Reynolds numbers. On the one hand, experimental mixing time is generally smaller than the simulated one and, on the other hand, no mixing time is determined by the CFD simulations for $\dot{V}=6 \mathrm{~mL} \mathrm{~min}^{-1}$. This deviation can be attributed to inaccuracies in the generation of the CAD model of the mixing structure. These inaccuracies result in differences between the real and the modeled structure, which can have a major impact on the flow behavior, especially in micro process engineering. Additionally, it is assumed that the limit of the mixing quality was set too high initially. Hence, the limit was adjusted to approximate CFD and experimental results. Based on Fig. 6b, the limit was adjusted to 0.8 to meet both of the aforementioned criteria. Figure 12 displays the adjusted CFD and experimental mixing times over energy dissipation rates.

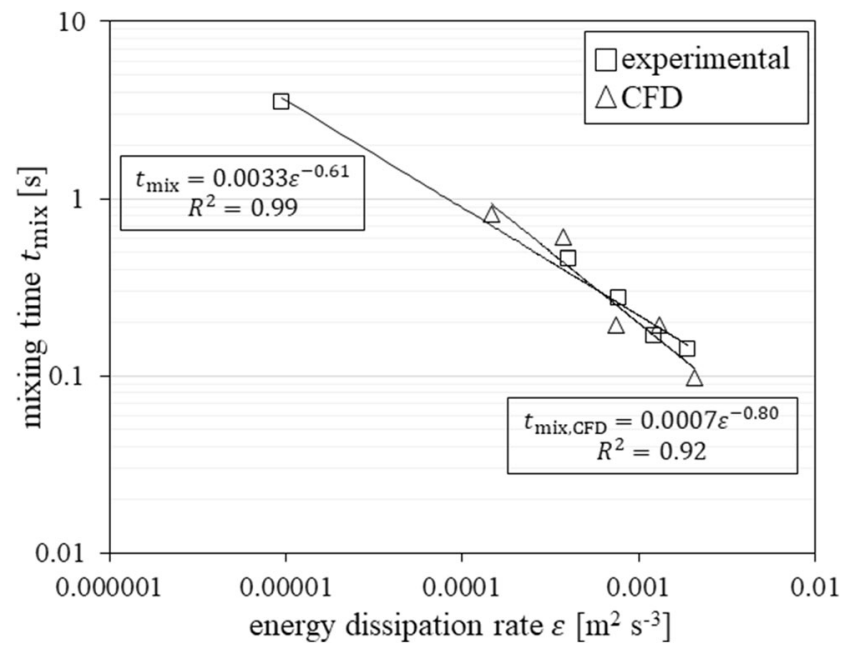

Fig. 12 Comparison of CFD and experimental mixing time over energy dissipation rates with adjusted limit for complete mixing of $\alpha^{\cdot}{ }_{V} \geq 0.8$.

By adjusting the limit, complete mixing is also achieved for 4 and $6 \mathrm{~mL} \mathrm{~min}^{-1}$ using CFD model. Since experimental and CFD results are generally in good agreement, the CFD model can be used to determine suitable experimental settings in the form of reasonable flow rates. Although the adjustment leads to a better description of the mixing, it can be observed that the exponent with a value of 0.8 deviates strongly from the literature value. This indicates that matching is not essential to derive mixing times from the numerical data that are sufficiently accurate for subsequent experimental design and that agree sufficiently well with theory.

\section{Case study: Experimental settings}

On the basis of the method used, optimal reaction conditions are translated into experimental settings. Therefore, the experimental design space is visualized using a parameter plot with volumetric flow rate and temperature as experimental parameter. The discrete step size for the parameters is set to $1 \mathrm{~mL} \mathrm{~min}{ }^{-1}$ for the volumetric flow rate and to $10^{\circ} \mathrm{C}$ for the temperature. The influence of temperature on the reaction time can be neglected, since the reaction is instantaneous and dominated by mixing. According to the presented methodology, which has been validated on the basis of experimental data, the experimental design space is located between volumetric flow rates of 6 and $12 \mathrm{~mL} \mathrm{~min}$. The limitation of the temperature $T_{\max }=90{ }^{\circ} \mathrm{C}$ is due to fact that aqueous solutions are used which start to boil for higher temperatures. This is a limitation that can be overcome, for example using a back-pressure regulator, but is certainly a limitation of the setup used in this study. This step needs to contain information about the currently used setup and suggestions to overcome arising 


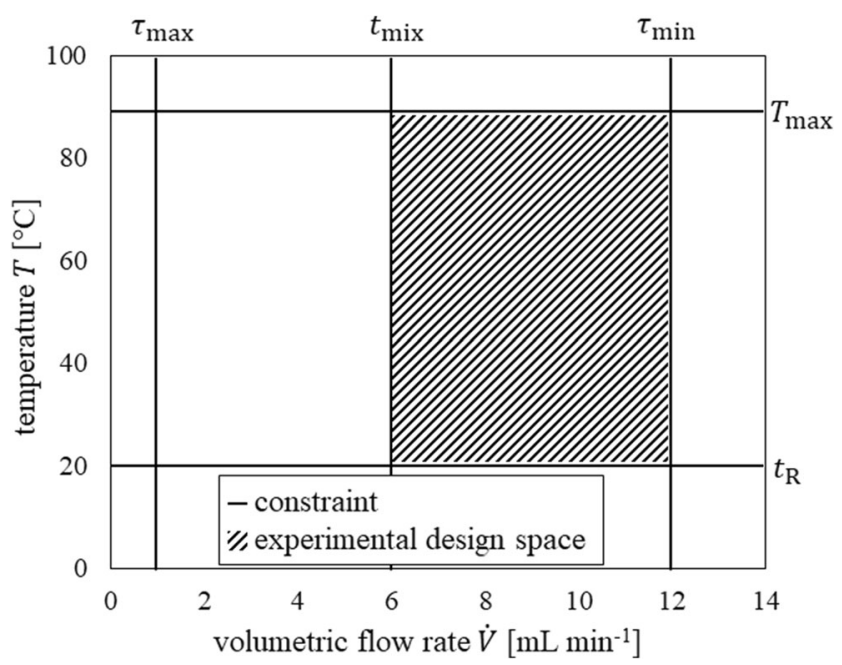

Fig. 13 Quantitative representation of the experimental design space for calorimetric measurements as function of temperature and volumetric flow rate

limitations be changing parts of the setup. As can be seen in Fig. 13, the experimental design space is halved by the methodology.

\section{Case study: Determination of thermokinetic data}

The reaction enthalpy of the neutralization reaction was determined at the previously determined experimental settings and compared to the literature value. According to Fig. 13, calorimetric measurements were performed at flow rates of 6 and $8 \mathrm{~mL} \mathrm{~min}^{-1}$ and at ambient temperature. In addition, a counter test with a flow rate of $4 \mathrm{~mL} \mathrm{~min}{ }^{-1}$ was investigated to demonstrate the applicability of the methodology. In Fig. 14, the heat flux profiles and the experimentally determined reaction enthalpies are displayed for varying volumetric flow rates.
For 6 and $8 \mathrm{~mL} \mathrm{~min}^{-1}$, a peak in the heat flux profile was observed indicating complete conversion of reactants. This is also confirmed by the good agreement of measured reaction enthalpy with the literature value. In contrast, no peak and thus no complete conversion was observed for $4 \mathrm{~mL} \mathrm{~min}^{-1}$, which can also be seen from the deviating reaction enthalpy. This result confirms the assumptions made previously and the applicability of the methodology.

\section{Conclusion and outlook}

In this study, a stepwise methodology to minimize the experimental effort for thermokinetic data acquisition was presented and evaluated. Short-cut calculations and CFD simulations using the open source software OpenFOAM were carried out to determine the residence, mixing and reaction time of a neutralization reaction within a commercially available microreactor. Additionally, the pressure drop as a technical criterion was determined. The results obtained showed good agreement with experimental results of pressure drop and mixing time. Thus, the CFD model was validated. However, deviations were observed, especially at low Reynolds numbers. Thus, an investigation with greater attention to diffusion would be necessary. The results of the methodology also enabled to minimize the possible volumetric flow rate by half. Consequently, the design space of experiments is rigorously reduced. The reasonable restriction of the design space was demonstrated by determination of reaction enthalpy, which resulted in meaningful data only for the proposed settings. In future studies, the input data set is to be extended by further commercially available microreactors in order to select a suitable reactor depending on the chemical reaction to be investigated. Hence, a tool for contributing expert
Fig. 14 a Spatially-resolved specific heat flux signals for neutralization reaction of $1 \mathrm{M}$ $\mathrm{HCl}$ and $1.1 \mathrm{M} \mathrm{NaOH}$ for varying volumetric flow rates. b Measured neutralization enthalpies for varying volumetric flow rates and comparison to literature value (dotted line at $57.6 \mathrm{~kJ} \mathrm{~mol}^{-1}$ )
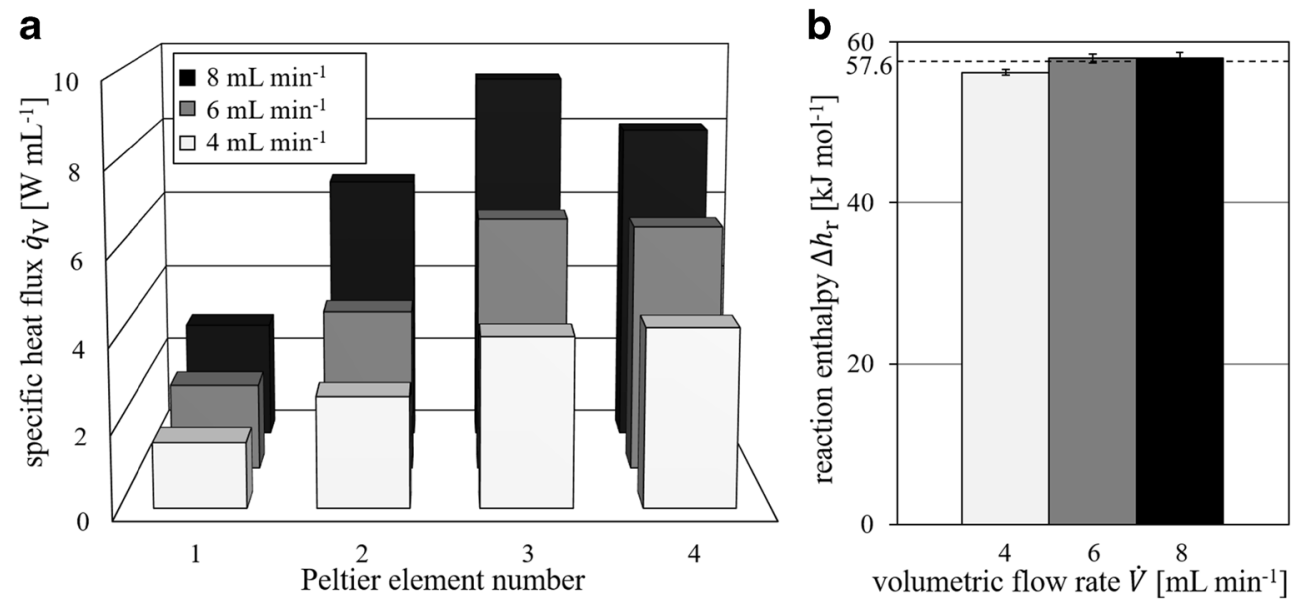
knowledge has to be created in the same step to reliably estimate the reaction time. Therefore, consistent ranges of parameters have to be set based on initial assumptions. In addition, the experimental parameter plots are to be extended by the concentration to counteract limitations by varying the inlet concentration of the reactants resulting in a three-dimensional design space. Besides modeling of hydrodynamics, the methodology should be supplemented via heat management to ensure suitable and safe reaction conditions. For this purpose, Westermann and Mleczko [36] proposed a short-cut approach that can be adapted to the presented methodology.

\begin{abstract}
Acknowledgements The German Federal Ministry of Economic Affairs and Energy (BMWi) is acknowledged for funding this research of the Industrielle Gemeinschaftsforschung (IGF, IGF project: 20819 N) which is organized by the Arbeitsgemeinschaft industrieller Forschungsvereinigung (AiF) and the Forschungs-Gesellschaft Verfahrenstechnik e.V. (GVT). The authors also gratefully acknowledge the computing time provided on the Linux HPC cluster at TU Dortmund University (LiDO3), partially funded in the course of the Large-Scale Equipment Initiative by the German Research Foundation (DFG) as project 271512359. Furthermore, we acknowledge financial support by German Research Foundation (DFG) and TU Dortmund University within the funding program Open Access Publishing.
\end{abstract}

Funding Open Access funding enabled and organized by Projekt DEAL.

Declarations The authors declare no competing financial interest.

Conflicts of interest/competing interests Not applicable.

Symbols $A_{\mathrm{M}}$, cross section area $\left[\mathrm{m}^{2}\right] ; c_{\mathrm{A}} 0$, starting concentration of limiting component $\mathrm{A}\left[\mathrm{mol} \mathrm{m}^{-3}\right] ; c_{\mathrm{i}}$, concentration at grid point $\mathrm{i}[\mathrm{mol}$ $\left.\mathrm{m}^{-3}\right] ; D$, diffusion coefficient $\left[\mathrm{m}^{2} \mathrm{~s}^{-1}\right] ; d_{\mathrm{c}}$, diameter of curvature $[\mathrm{m}] ; d_{\mathrm{h}, \mathrm{i}}$, hydraulic diameter $[\mathrm{m}] ; E_{\mathrm{A}}$, activation energy $\left[\mathrm{J} \mathrm{mol}^{-1}\right] ; h_{\mathrm{r}}$, molar reaction enthalpy $\left[\mathrm{J} \mathrm{mol}^{-1}\right] ; k\left(T_{\mathrm{R}}\right)$, rate constant $[-] ; k_{0}$, pre-exponential factor $\left[\mathrm{m}^{3}\right.$ $\left.\mathrm{mol}^{-1} \mathrm{~s}^{-1}\right] ; l_{\mathrm{i}}$, channel length $[\mathrm{m}] ; m$, reaction order [-]; $n$, exponent of power law trend line [-]; $p$, pressure $[\mathrm{Pa}] ; R^{2}$, coefficient of determination $[-] ; t_{\text {mix }}$, , mixing time $[\mathrm{s}] ; t_{\text {mix }}$ CFD , mixing time from simulation [s]; $T$, temperature $[\mathrm{K}] ; T_{\max }$, maximum temperature $[\mathrm{K}] ; T_{\mathrm{R}}$, reactor temperature $[\mathrm{K}] ; \cdot V$, volumetric flow rate $\left[\mathrm{m}^{3} \mathrm{~s}^{-1}\right] ; V_{\mathrm{i}}$, dissipation volume $\left[\mathrm{m}^{3}\right]$; $V_{\mathrm{R}}$, reactor volume $\left[\mathrm{m}^{3}\right] ; \bar{w}$, averaged velocity $\left[\mathrm{m} \mathrm{s}^{-1}\right] ; w_{\mathrm{i}}$, velocity $\left[\mathrm{m} \mathrm{s}^{-1}\right]$

Dimensionless numbers $\quad D n$, Dean number [-]; Re, Reynolds number [-]

Greek symbols $\alpha \cdot V$, velocity-weighted mixing quality [-]; $\Delta$, difference [-]; $\varepsilon$, energy dissipation rate $\left[\mathrm{m}^{2} \mathrm{~s}^{-3}\right] ; \lambda$, friction factor $[-] ; \bar{\nu}$, kinematic viscosity $\left[\mathrm{m}^{2} \mathrm{~s}^{-1}\right] ; \bar{\rho}$, density $\left[\mathrm{kg} \mathrm{m}^{-3}\right] ; \sigma_{\mathrm{V}}^{2}$, variance at cross section [-]; $\sigma_{\text {V,max }}^{2}$, maximum variance at inlet [-]; $\tau$, residence time [s]; $\tau_{\min }$, minimum residence time $[\mathrm{s}] ; \tau_{\max }$, maximum residence time $[\mathrm{s}] ; \omega_{\mathrm{i}}$, influence of secondary flow patterns [-]

Abbreviations CFD, computational fluid dynamics; HCL, hydrochloric acid; $\mathrm{NaOH}$, sodium hydroxide; OPC UA, Open Platform Communications Unified Architecture; PLC, programmable logic controller
Open Access This article is licensed under a Creative Commons Attribution 4.0 International License, which permits use, sharing, adaptation, distribution and reproduction in any medium or format, as long as you give appropriate credit to the original author(s) and the source, provide a link to the Creative Commons licence, and indicate if changes were made. The images or other third party material in this article are included in the article's Creative Commons licence, unless indicated otherwise in a credit line to the material. If material is not included in the article's Creative Commons licence and your intended use is not permitted by statutory regulation or exceeds the permitted use, you will need to obtain permission directly from the copyright holder. To view a copy of this licence, visit http://creativecommons.org/licenses/by/4.0/.

\section{References}

1 McMullen JP, Stone MT, Buchwald SL, Jensen KF (2010) Angew Chem Int Ed 49(39):7076-7080. https://doi.org/10.1002/anie. 201002590

2 Heitmann D (2016) Chem Eng Technol 39(11):1993-1995. https:// doi.org/10.1002/ceat.201600150

3 Elvira KS, Casadevall i Solvas X, Wootton RCR, deMello AJ (2013) Nat Chem 5(11):905-915. https://doi.org/10.1038/nchem. 1753

4 Litz W (2015) Bench scale calorimetry in chemical reaction kinetics: An alternative approach to liquid phase reaction kinetics, 1st edn. Springer, Cham

5 Mortzfeld F, Polenk J, Guelat B, Venturoni F, Schenkel B, Filipponi P (2020) Org Process Res Dev 24(10):2004-2016. https://doi.org/ 10.1021/acs.oprd.0c00117

6 Ładosz A, Kuhnle C, Jensen KF (2020) React Chem Eng 5(11): 2115-2122. https://doi.org/10.1039/D0RE00304B

7 Hosoya M, Nishijima S, Kurose N (2020) Org Process Res Dev 24(6):1095-1103. https://doi.org/10.1021/acs.oprd.0c00109

8 Maier MC, Leitner M, Kappe CO, Gruber-Woelfler H (2020) React Chem Eng 5(8):1410-1420. https://doi.org/10.1039/D0RE00122H

9 Kashid MN, Renken A, Kiwi-Minsker L (2015) Microstructured devices for chemical processing. Wiley-VCH, Weinheim

10 Asprion N, Böttcher R, Mairhofer J, Yliruka M, Höller J, Schwientek J, Vanaret C, Bortz M (2020) J Chem Eng Data 65(3):1135-1145. https://doi.org/10.1021/acs.jced.9b00494

11 Yang L, Nieves-Remacha MJ, Jensen KF (2017) Chem Eng Sci 169:106-116. https://doi.org/10.1016/j.ces.2016.12.003

12 de Sousa MR, Santana HS, Taranto OP (2020) Int J Multiph Flow 132:103435. https://doi.org/10.1016/j.ijmultiphaseflow.2020. 103435

13 Asano S, Yatabe S, Maki T, Mae K (2019) Org Process Res Dev 23(5):807-817. https://doi.org/10.1021/acs.oprd.8b00356

14 Reichmann F, Millhoff S, Jirmann Y, Kockmann N (2017) Chem Eng Technol 40(11):2144-2154. https://doi.org/10.1002/ceat. 201700419

15 Reichmann F, Vennemann K, Frede TA, Kockmann N (2019) Chem Ing Tech 91(5):622-631. https://doi.org/10.1002/cite. 201800169

16 Krasberg N, Hohmann L, Bieringer T, Bramsiepe C, Kockmann N (2014) Processes 2(1):265-292. https://doi.org/10.3390/pr2010265

17 Knösche CM (2005) Chem Ing Tech 77(11):1715-1722. https://doi. org/10.1002/cite.200500123

18 Deen WM (2012) Analysis of transport phenomena, 2nd ed., Topics in chemical engineering. Oxford University Press, New York

19 Holvey CP, Roberge DM, Gottsponer M, Kockmann N, Macchi A (2011) Chem Eng Process 50(10):1069-1075. https://doi.org/10. 1016/j.cep.2011.05.016

20 Dean WR (1928) Proc R Soc Lond A 121(787):402-420. https:// doi.org/10.1098/rspa.1928.0205 
21 Hessel V, Löwe H, Schönfeld F (2005) Chem Eng Sci 60(8-9): 2479-2501. https://doi.org/10.1016/j.ces.2004.11.033

22 Microfluidics (2011) In: Lin B, Basuray S (eds) Technologies and applications, Topics in current chemistry. Springer, Berlin

23 Kockmann N (ed) (2006) Micro process engineering, vol 5. Wiley$\mathrm{VCH}$, Weinheim

24 Howell PB, Mott DR, Golden JP, Ligler FS (2004) Lab Chip 4(6): 663-669. https://doi.org/10.1039/b407170k

25 Kumar V, Aggarwal M, Nigam K (2006) Chem Eng Sci 61(17): 5742-5753. https://doi.org/10.1016/j.ces.2006.04.040

26 Bałdyga J, Bourne JR (1999) Turbulent mixing and chemical reactions. Wiley-VCH, New York

27 Falk L, Commenge J-M (2010) Chem Eng Sci 65(1):405-411. https://doi.org/10.1016/j.ces.2009.05.045

28 Kockmann N (2008) Transport phenomena in micro process engineering. Springer, Berlin

29 Roberge DM (2004) Org Process Res Dev 8(6):1049-1053. https:// doi.org/10.1021/op0400160

30 Sen MA, Kowalski GJ, Fiering J, Larson D (2015) Thermochim Acta 603:184-196. https://doi.org/10.1016/j.tca.2014.09.024
31 Lide DR (2004) CRC handbook of chemistry and physics: A readyreference book of chemical and physical data, 85th edn. CRC Press Taylor \& Francis, Boca Raton

32 Ligrani PM (1994) A study of dean vortex development and structure in a curved rectangular channel with aspect ratio of 40 at dean numbers up to 430. NASA Contractor Report 4607

33 Fischer M, Kockmann N (2012) In: Proc. of ASME 12th Int. Conference on Nanochannels, Microchannels, and Minichannels 2012, American Society of Mechanical Engineers, Puerto Rico

34 Khaydarov V, Borovinskaya E, Reschetilowski W (2018) Appl Sci 8(12):2458. https://doi.org/10.3390/app8122458

35 Mortimer CE, Müller U (2020) Chemie: das basiswissen der chemie. Georg Thieme Verlag, Stuttgart

36 Westermann T, Mleczko L (2016) Org Process Res Dev 20(2):487494. https://doi.org/10.1021/acs.oprd.5b00205

Publisher's note Springer Nature remains neutral with regard to jurisdictional claims in published maps and institutional affiliations. 